\title{
Evaluating influence of the genotypes in the follicle-stimulating hormone receptor (FSHR) Ser680Asn (rs6166) polymorphism on poor and hyper-responders to ovarian stimulation: a meta-analysis
}

Noel Pabalan ${ }^{1,2,3}$, Camila Martins Trevisan ${ }^{4}$, Carla Peluso ${ }^{4}$, Hamdi Jarjanazi ${ }^{5}$, Denise Maria Christofolini ${ }^{4}$, Caio Parente Barbosa ${ }^{4}$ and Bianca Bianco ${ }^{4 *}$

\begin{abstract}
Background/aims: Reported associations of controlled ovarian hyperstimulation response $(\mathrm{COH})$ with genotypes of the Ser680Asn (N680S) polymorphism in the follicle stimulating hormone receptor (FSHR) gene have conflicting results.

Methods: PubMed and Embase databases were searched for studies that investigated the N680S polymorphism in the FSHR gene in $\mathrm{COH}$. Parameters used to examine ovarian response were poor and hyper-responses to $\mathrm{COH}$. Using the meta-analytic approach, we estimated ovarian response risk (odds ratio [OR] with 95\% confidence intervals) according to genotype.
\end{abstract}

Results: Our findings showed that SS genotype carriers were most likely to be poor responders (OR 1.61, $p=0.08$ ) compared to the NN and NS genotypes which showed no associations (OR 0.93-0.95, $p=0.75-0.78$ ). Heterogeneity of these pooled ORs warranted examining its sources. We detected outlying studies in each of the three N680S genotypes. Omitting these outliers erased the heterogeneity of the recalculated pooled outcomes. It also materially altered the SS effects where carriers became slightly unlikely to be poor responders (OR 0.90, $p=0.52$ ). The $S$ allele carrier effect was modulated for poor responders (OR 1.24, $p=0.39)$ in the Non-Hispanic Caucasian (NHC) subgroup. The likelihood of the $S$ allele carriers (OR 1.47, $p=0.02$ ) and the unlikelihood of the $N$ allele carriers (OR 0.64, $p=0.007$ ) were significant in our hyper-response findings. Confined to NHC retained significance of the $S$ allele effects (OR 1.57, $p=0.01$ ) but not among the $N$ allele carriers (OR 0.68, $p=0.18$ ).

Conclusions: In summary, this is a meta-analytical confirmation of the FSHR SS genotype role in COH response. Hyper-responder analysis strengths lie on the non-heterogeneity and robustness of its results. Non-robustness and heterogeneity of the poor-responder results compose its limitations. Thus, poor response findings probably require caution as to the interpretation as a susceptibility marker for ovarian response.

Keywords: Follicle-stimulating hormone receptor, FSHR, Polymorphisms, Ovarian response, Meta-analysis

\footnotetext{
* Correspondence: bianca.bianco@hotmail.com

${ }^{4}$ Human Reproduction and Genetics Center, Department of Collective Health -

Faculdade de Medicina do ABC, Av. Prncipe de Gales, 821, Santo Andr/SP,

So Paulo Zip Code 09060-650, Brazil

Full list of author information is available at the end of the article
} 


\section{Background}

In vitro fertilization (IVF) is a multi-step process involving collection of oocyte-containing follicles after controlled ovarian hyperstimulation $(\mathrm{COH})$ with $\mathrm{rFSH}$ (recombinant Follicle-stimulation hormone). Further steps involve oocyte fertilization, embryo development, embryo transfer to the uterus, and implantation. All these steps are critical for successful IVF [1]. An initial critical step of this complex procedure is the $\mathrm{COH}$, aiming to safely obtain an adequate number of high-quality oocytes, so as to allow selection of the most viable embryo for transfer $[1,2]$. However, women submitted to this procedure yield different numbers of oocytes. Poor responders provide no more than 4-5 oocytes. Those with 6-15 oocytes are normal responders and those with $>15$ oocytes are hyperresponders $[3,4]$. Poor responses result in retrieved oocytes reduced number and hyper-response may lead to ovarian hyperstimulation syndrome (OHSS). Poor response may warrant repeated stimulation cycles to obtain appropriate number of oocytes. In contrast, good response is modulated to avoid hyperstimulation [5]. Thus, the patients advanced identification will elicit these responses in order to standard treatment, which would be clinically beneficial. Several factors have been proposed to predict ovarian response. These are: age [6], hormonal status [7], cigarette smoking [8] and ovarian reserve [6,9]. Apart from these proposed predictors, polymorphisms in various genes, such as estrogen receptor alpha (ESR1), cytochrome P450 19A (CYP19A) and follicle-stimulating hormone (FSH) have been investigated as markers to predict ovarian response [1,10-13]. FSH has been implicated in follicular growth and maturation, granulosa cell proliferation and in estradiol/aromatase synthesis [14]. FSH effects are mediated by FSH receptor (FSHR), a G-protein-coupled receptor expressed in granulosa cells [15] which mediates FSH signal transduction through adenylate cyclase activation and elevation of intracellular cAMP [16]. The FSHR gene is located on chromosome 2p21 spanning a region of $54 \mathrm{~kb}$ [17] and contains one large exon, which encodes the transmembrane and intracellular domains; and nine smaller exons, which encode the extracellular domain [15]. Two non-synonymous SNPs have been identified in the coding region of exon 10 of the FSH receptor gene (http://www.ncbi.nlm.nih.gov/snp/?term=FSHR; GeneID: 2492; Locus tag: HGNC: 3969). The first (rs6165) is found within the extracellular domain (codon 307) in which A is substituted by $G$, changing codon 307 from threonine (ACT) to alanine (GCT). The second (rs6166) lies within the intracellular domain (codon 680), in which $G$ is replaced by $A$. This leads to an amino acid change at position 680 from serine (AGT) to asparagine (AAT) [18]. These two SNPs are related to ovarian response and affect gene function by changing the e gene product properties and consequently modifying response to FSH [19]. These polymorphisms are in linkage disequilibrium (LD), resulting in the most frequent allelic combination of T307-N680 and A307-S680 [20,21]. Many previous studies including a recent meta-analysis [22] focused on the N680S polymorphism. Clinical studies have demonstrated that the N680S polymorphism determines ovarian response to FSH stimulation in patients undergoing IVF treatment $[11,22,23]$.

Given the conflicting outcomes in the human reproduction investigations of the variants in the FSHR gene $[17,24]$, we performed a meta-analysis to evaluate the role of the N680S FSHR polymorphism in ovarian response. Here, we compared the association of inactivating FSHR genotypes in (i) poor and (ii) hyperresponding women with normal ovarian response.

\section{Materials and methods}

\section{Selection of studies}

Using the terms, FSHR, follicle stimulating hormone, polymorphism and ovarian stimulation, we searched MEDLINE using PubMed and Embase for associated studies as of September 12, 2014. References cited in the retrieved articles were also screened manually to identify additional eligible studies. Inclusion criteria included: (1) case control study design evaluating the association between FSHR polymorphisms and ovarian response, (2) sufficient genotype frequency data presented to calculate the odds ratios (ORs) and 95\% confidence intervals (CIs). (3) Control frequencies (normal/good responders) must be in Hardy-Weinberg Equilibrium (HWE).

\section{Data extraction}

Two investigators, independently, extracted data and reached consensus on all the items. The following information was obtained from each publication: first author s name, published year, country of origin, dominant ancestry of the study populations, study design, context of the study, type of ovarian response, use of the HWE, addressed LD, FSHR polymorphism studied, sample source, genotyping approach, matching information and genotype data. We extracted data that belonged to our investigation of ovarian response using these parameters: (i) number of poor and (ii) hyperresponders as well as (iii) normal (good/control) responders. We also calculated frequencies of the variant allele, deviations of the normal responders from the HWE. (iv) Rates of pregnancy.

\section{Quality assessment of the studies}

We used the Clark-Baudouin Score (CBS) to evaluate the methodological quality of the included studies [25]. This scale emphasizes statistics (i.e. p values, power and corrections for multiplicity) and includes genotyping 
methods as well as HWE. These, among others, were addressed in several of the included papers. Thus, we felt this to be the most appropriate in assessing the methodological included studies quality. The CBS scores reach from 0 (worst) to 9 (best) for cohort studies and 0 (worst) to 10 (best) for case-control studies. The score follows as: case-control studies: (quality is low for $<5$ and high for $\geq 5$ ); cohort studies: (quality is low for $<4$ and high for $\geq 4$ ).

\section{Meta-analysis}

Due to the FSHR polymorphisms (T307A and N680S) were found to be in complete LD $[20,21]$, we only investigated the N680S polymorphism. Influence of FSHR N680S polymorphism was estimated using the following parameters: we examined (i) poor ovarian response, (ii) hyper-response compared to the (iii) normal or good responders using the homozygote variant and wildtype alleles as well as the heterozygote genotype. We also examined (iv) pregnancy rates. These associations were expressed as OR, 95\% CI.

Raw data for genotype frequencies, without adjustment, were used for calculating OR study-specific estimates. The pooled estimates significance was determined by the Z-test. Pooled estimates were obtained using either the fixed [26] (in the absence of heterogeneity) or random [27] (in its presence) effects models. Heterogeneity between studies was estimated using the $\chi^{2}$-based $Q$ test [28]. Recognizing the low power of this test [29], significance threshold was set at $\mathrm{p}=0.10$. We also quantified heterogeneity with the $\mathrm{I}^{2}$ statistic which measures the degree of inconsistency among studies [30]. Significance was set at a p-value of $\leq 0.05$ throughout, except in heterogeneity estimation. Pooled estimates were submitted to sensitivity analysis which involved omitting one study at a time and recalculating the pooled estimates, to test the summary effect robustness. Subgroup analysis based on ethnicity was performed on those comprising of three or more subgroups. Data were analyzed using Review Manager 5.3 (Copenhagen: Nordic Cochrane Centre, Cochrane Collaboration, 2014) and SigmaStat 2.3 (Systat Software, San Jose, CA). Most comparisons in our meta-analysis had less than 10 studies, and because of the low sensitivity of the qualitative and quantitative tests [31], we did not investigate publication bias. However, the overall analysis of poor responders had 11 studies; for those, we used Beggs test to evaluate whether the magnitude of the observed association was related to the variance of each study [32].

\section{Results}

\section{Included studies}

Figure 1 outlines our study selection process in a flowchart following PRISMA (Preferred Reporting Items for
Systematic Reviews and Meta-Analyses) guidelines [33]. We identified a total of 215 citations during the initial search, from which 161 were omitted because they were not conforming our inclusion criteria. We retrieved the remaining 54 abstracts which after review, five articles were excluded. Full-texts of the remaining 49 were obtained and read including the reference lists. From these lists, we found five articles with potential inclusion in the meta-analysis. From the 54 full-text articles, 43 were removed, including two studies [3,34] which control frequencies deviated from the HWE. Under these circumstances, the total number of articles included in the meta-analysis was 11 [1,4,13,35-42]. This number was increased to 13 studies, due to the provision of the independent data added from Boudjenah et al [1] and Livshyts et al [39].

The epidemiological features and clinical characteristics of these studies are outlined in Table 1. The publication years of the included studies ranged from 2003 to 2014. Six studies [1,36,38-40,42] had subjects that were of non-Hispanic Caucasian (NHC) descendant, two $[13,37]$ were Hispanic Caucasian $(\mathrm{HC})$ and three were Asian [4,35,41]. Eight of each 11 articles (72.7\%) addressed HWE and LD, respectively. Based on the CBS scores, methodological quality of the included papers was high (mean and standard deviation of 5.82 ? 1.3).

Additional file 1: Table S1 shows the 11 studies from nine articles [1,4,13,35,37-42] summarizing the genotype frequencies of the poor responders compared to normal/ good responders. Additional file 2: Table S2 displays the six studies from five articles $[1,13,35,36,40]$ summarizing genotype frequencies of the hyper-responders compared to normal responders.

\section{Overall and subgroup analysis}

We found no evidence of publication bias in the overall analysis of poor responders $(\mathrm{p}=0.39-0.94)$. Table 2 and Figures 2 and 3 show an overall favoring of the SS genotype among the ovarian response groups, significant for hyper-responders (OR 1.47, $\mathrm{p}=0.02$ ) but not for poor responders (OR 1.61, $\mathrm{p}=0.08$ ). Confined to NHC modulated the poor responder effects (OR 1.24, $\mathrm{p}=0.39)$ but not the hyper-responder effects (OR $1.57, \mathrm{p}=0.01)$. The $\mathrm{N}$ allele carriers unlikelihood of being ovarian responders was exemplified among hyper-responders with an overall significance (OR 0.64, $\mathrm{p}=0.007$ ). Of the studies listed in Table 1, six reported pregnancy rates $[3,34,36,38,40,43]$, half of these had controls that deviated from the HWE $[3,34,36]$. All pregnancy rates pooled effects indicated absence of associations (data not shown).

\section{Outlier analysis}

We sought outlier studies as sources of the heterogeneity observed in the overall poor responder pooled effects 


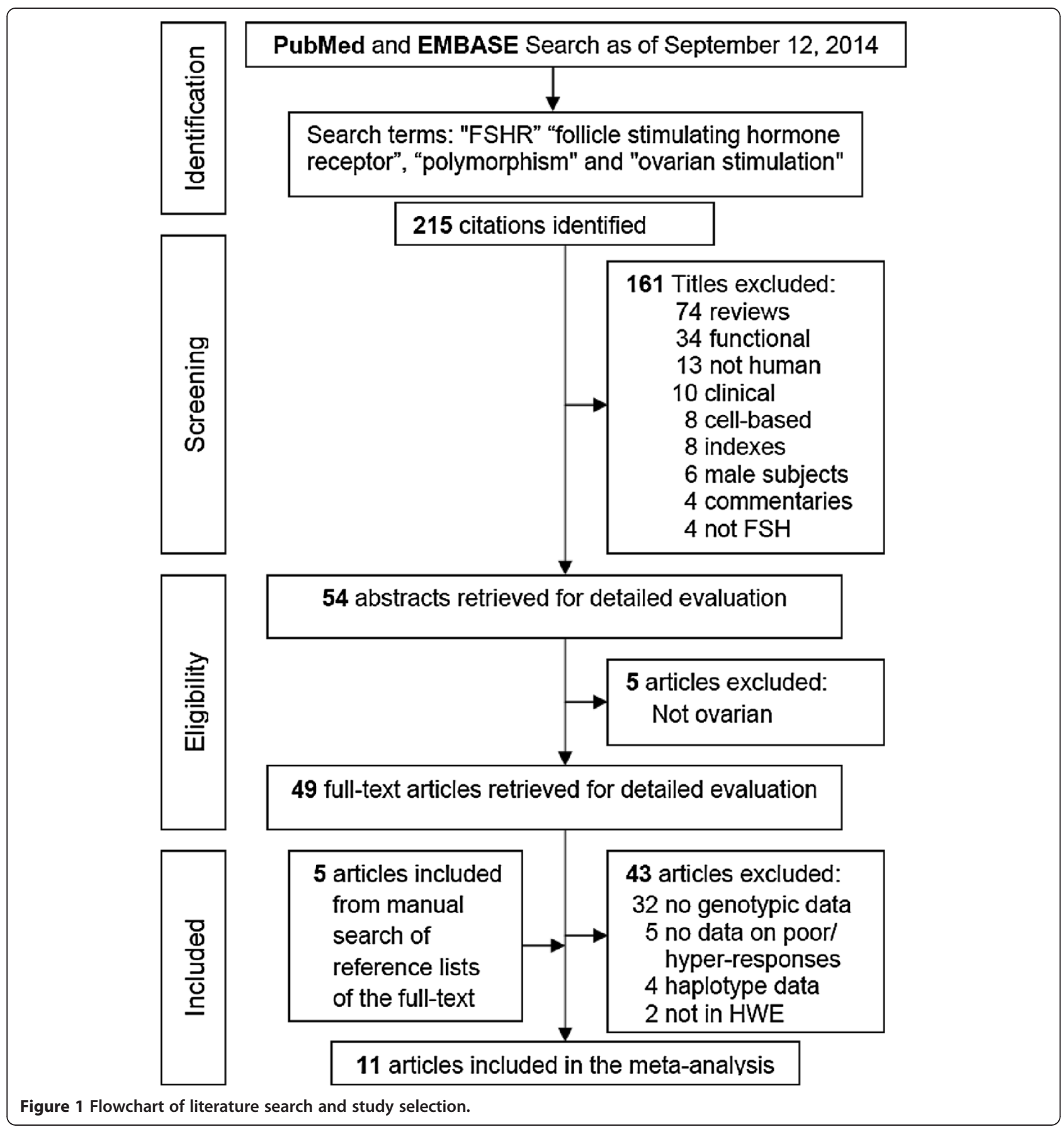

(Figure 2). The Galbraith plots in Figure 4 show the outliers, four studies from three articles [4,39,41] in the $\mathrm{S}$ allele, three studies $[37,39,41]$ in the $\mathrm{N}$ allele and one study [4] in the NS genotype. Table 2 shows the results of removing these outliers followed by re-analysis which erased heterogeneity ( $p$ values for heterogeneity from $<0.0001$ to $=0.78)$ in all three genotypes and altered the SS genotype effect (OR 0.90, p = 0.52).
Sensitivity analysis

Table 3 summarizes the changes in association resulting from sensitivity treatment. These changes have affected the poor responder group more than the hyper-responders. Generally, finding of no associations have been altered to the likelihood of $\mathrm{N}$ allele carriers to be poor responders with omission of three studies from two articles [39,41]. This pattern was also observed in hyper-responders where 
Table 1 Included articles characteristics that examined the FSHR N680S polymorphism association with ovarian response

\begin{tabular}{|c|c|c|c|c|c|c|c|c|c|c|c|}
\hline First author & PY & {$[\mathrm{R}]$} & Country & Ethnic group & Sample size & Type of ovarian response & Used HWE & Addressed LD & Tissue source & Genotyping & CBS \\
\hline Daelemans & 2004 & {$[36]$} & Belgium & $\mathrm{NHC}$ & 229 & Hyper/controls & No & Yes & blood & DNA sequencing & 4 \\
\hline Klinkert & 2006 & [38] & Nether-lands & $\mathrm{NHC}$ & 105 & Poor, not poor & No & Yes & blood & Taqman & 5 \\
\hline Livshyts & 2009 & [39] & Ukraine & $\mathrm{NHC}$ & 374 & poor, good/controls & Yes & Yes & blood & PCR-RFLP & 5 \\
\hline Boudjenah & 2012 & [1] & France & $\mathrm{NHC}$ & 427 & poor, hyper/controls & Yes & Yes & blood & Multiplex PCR & 7 \\
\hline Binder & 2012 & {$[42]$} & Germany & $\mathrm{NHC}$ & 259 & low, control & No & No & blood & RT-QPCR & 6 \\
\hline Mohiyiddeen & 2013 & {$[40]$} & United Kingdom & $\mathrm{NHC}$ & 504 & poor, normal, hyper & Yes & Yes & blood & Taqman & 5 \\
\hline de Castro & 2003 & [37] & Spain & $\mathrm{HC}$ & 102 & poor, good & Yes & Yes & blood & RT-QPCR & 5 \\
\hline de Castro & 2004 & [13] & Spain & $\mathrm{HC}$ & 170 & poor, rest of patients, high & Yes & No & blood & RT-QPCR & 8 \\
\hline Huang & 2014 & [4] & China & Asian & 1,250 & Poor, good responders & Yes & Yes & blood & MALDI-TOF & 8 \\
\hline Achrekar & 2009 & [35] & India & Asian & 150 & Hyper, not hyper & Yes & No & blood & RT-QPCR & 5 \\
\hline Yan & 2013 & [41] & China & Asian & 450 & Poor, not poor, hyper, not hyper & Yes & Yes & blood & RT-QPCR & 6 \\
\hline
\end{tabular}

PY: Publication Year; [R]: Reference; NHC: Non-Hispanic Caucasian; HC: Hispanic Caucasian; HWE: Hardy-Weinberg Equilibrium; LD: Linkage Disequilibrium PCR: Polymerase Chain Reaction; RFLP: Restriction Fragment Length Polymorphism; RT-QPCR: Real-Time Quality Polymerase Chain Reaction; MALDI-TOF: Matrix Assisted Laser Desorption lonization-Time-of-Flight; CBS: Clark-Baudouin Score: assessment of methodological quality of the included studies. 
Table 2 Summary of the FSHR N680S polymorphism with poor and hyper-responders

\begin{tabular}{|c|c|c|c|c|c|c|c|c|c|c|c|c|c|c|c|c|c|c|c|}
\hline & \multirow[b]{3}{*}{$\mathbf{N}$} & \multicolumn{6}{|c|}{$\mathrm{S}$ allele } & \multicolumn{6}{|c|}{$\mathrm{N}$ allele } & \multicolumn{6}{|c|}{ NS genotype } \\
\hline & & \multicolumn{3}{|c|}{ Test of association } & \multicolumn{3}{|c|}{ Test of heterogeneity } & \multicolumn{3}{|c|}{ Test of association } & \multicolumn{3}{|c|}{ Test of heterogeneity } & \multicolumn{3}{|c|}{ Test of association } & \multicolumn{3}{|c|}{ Test of heterogeneity } \\
\hline & & OR & $95 \% \mathrm{Cl}$ & $\mathrm{pA}$ & $\mathrm{pB}$ & $I^{2}$ & $A M$ & OR & $95 \% \mathrm{Cl}$ & $\mathrm{pA}$ & $\mathrm{pB}$ & $1^{2}$ & $\mathrm{AM}$ & OR & $95 \% \mathrm{Cl}$ & pA & $\mathrm{pB}$ & $I^{2}$ & $A M$ \\
\hline \multicolumn{20}{|l|}{ Poor responders } \\
\hline All & 11 & 1.61 & $0.94-2.74$ & 0.08 & $<0.0001$ & 80 & $\mathrm{R}$ & 0.95 & $0.66-1.37$ & 0.78 & 0.002 & 65 & $\mathrm{R}$ & 0.93 & $0.60-1.46$ & 0.75 & $<0.0001$ & 80 & R \\
\hline Outliers omitted & & 0.90 & $0.66-1.23$ & $0.52^{*}$ & 0.16 & 36 & $\mathrm{~F}$ & 1.02 & $0.82-1.26$ & $.88^{* *}$ & 0.10 & 42 & $\mathrm{~F}$ & 0.82 & $0.66-1.02$ & $.08^{* * *}$ & 0.78 & 0 & $\mathrm{~F}$ \\
\hline $\mathrm{NHC}$ & 7 & 1.24 & $0.76-2.05$ & 0.39 & 0.03 & 57 & $\mathrm{R}$ & 0.99 & $0.60-1.62$ & 0.97 & 0.009 & 65 & $\mathrm{R}$ & 0.88 & $0.69-1.13$ & 0.32 & 0.79 & 0 & $\mathrm{~F}$ \\
\hline \multicolumn{20}{|l|}{ Hyper-responders } \\
\hline All & 6 & 1.47 & $1.05-2.04$ & 0.02 & 0.23 & 28 & $\mathrm{~F}$ & 0.64 & $0.46-0.88$ & 0.007 & 0.19 & 32 & $\mathrm{~F}$ & 1.10 & $0.83-1.46$ & 0.50 & 0.37 & 7 & $\mathrm{~F}$ \\
\hline $\mathrm{NHC}$ & 4 & 1.57 & $1.11-2.23$ & 0.01 & 0.33 & 13 & $\mathrm{~F}$ & 0.68 & $0.38-1.20$ & 0.18 & 0.07 & 57 & $\mathrm{R}$ & 1.03 & $0.76-1.40$ & 0.83 & 0.69 & 0 & $\mathrm{~F}$ \\
\hline
\end{tabular}




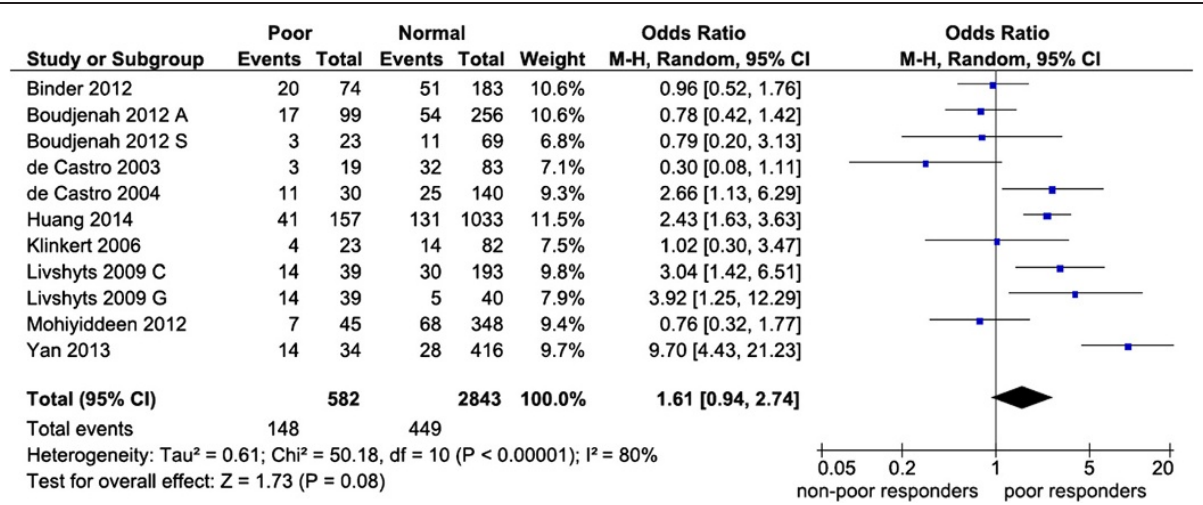

Figure 2 Forest plot of the $\mathbf{S} 680$ influence variant on poor ovarian response. Black diamond denotes the pooled OR. Blue squares indicate the OR in each study, with square sizes directly proportional to the weight contribution (\%) of the study. Horizontal lines represent $95 \%$ confidence intervals. Note: In Boudjenah et al. [1], the suffixes A and S indicate overall population and homogeneous subgroup, respectively; In Livshyts 2009, the suffixes $C$ and $\mathrm{G}$ indicate control women under 35 years old and over 35 years old, respectively. M-H: Mantel-Haenszel; Cl: confidence interval; df: degree of freedom.

no associations were changed to likelihood of NS carriers to be non-hyper-responders with omission of Daelemans et al [36].

\section{Discussion}

With a sample size of 4,020 for the N680S FSHR polymorphism, our meta-analysis has shown that the FSHR gene genotype is important in determining ovarian response. The N680S polymorphism has been shown to be associated with poor and hyper ovarian responses to stimulation. From the three genotypes, the most and least likely to be poor and hyper-responders to ovarian stimulation are SS and NS carriers, respectively. SS genotype carriers favoring seem to merit higher confidence in the hyper-responder findings than those in poor responders for two reasons: (i) hyper-responder findings were generally not heterogeneous which the poor responder results were. (ii) Sensitivity treatment showed that poor responder effects were not as robust as those of the hyper-responders. To our knowledge, this is the first meta-analysis to address poor and hyperresponses as indicators of ovarian response. A recent meta-analysis [22] examined the number of retrieved oocytes and basal FSH levels based on eight studies, but not the parameters we used here, except pregnancy rates in which our findings did not materially differ.

The study-specific findings showed that women homozygous for the FSHR S680 variant were less likely to be low responders and more likely to be high responders [1]. In a Chinese study of infertile women, the polymorphisms T307A and N680S were associated with ovarian response to $\mathrm{FSH}$, with the $\mathrm{SS}$ genotype having higher rates of poor response, but not with OHSS [41]. A retrospective study in IVF patients has shown an association between the presence of the $S$ variant and poor responses to gonadotropin stimulation, suggesting that the S680 allele was associated with a diminished sensitivity to FSH [37]. The implication of S680 allele as a potential marker for predicting poor ovarian response has been countered with reports suggesting no N680S association with ovarian response [44]. It has been suggested that the subjects with the NS genotype are more associated with good response to FSH stimulation, whereas the subjects with SS and NN genotypes have a

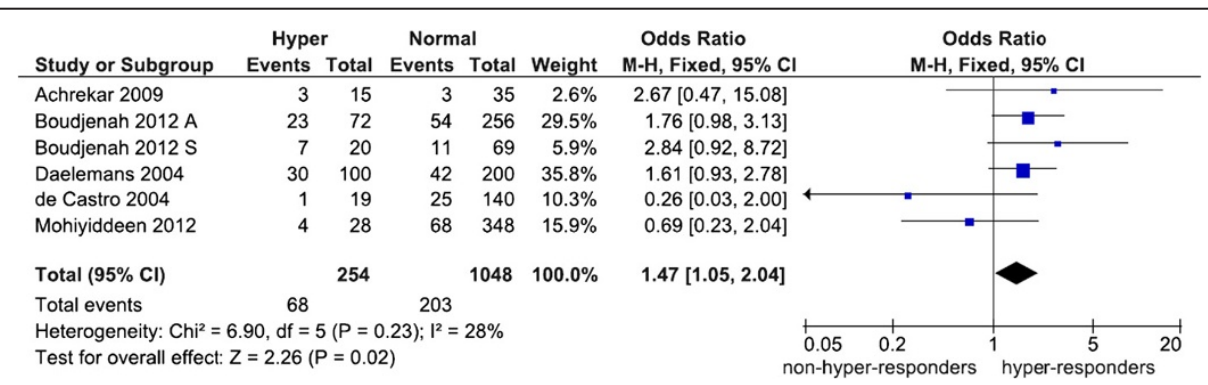

Figure 3 Forest plot of the $\mathbf{S 6 8 0}$ influence variant on hyper ovarian response. Black diamond denotes the pooled OR. Blue squares indicate the OR in each study, with square sizes directly proportional to the weight contribution (\%) of the study. Horizontal lines represent $95 \%$ confidence intervals. Note: In Boudjenah et al. [1], the suffixes A and S indicate overall population and homogeneous subgroup, respectively; . M-H: Mantel-Haenszel; Cl: confidence interval; df: degree of freedom. 


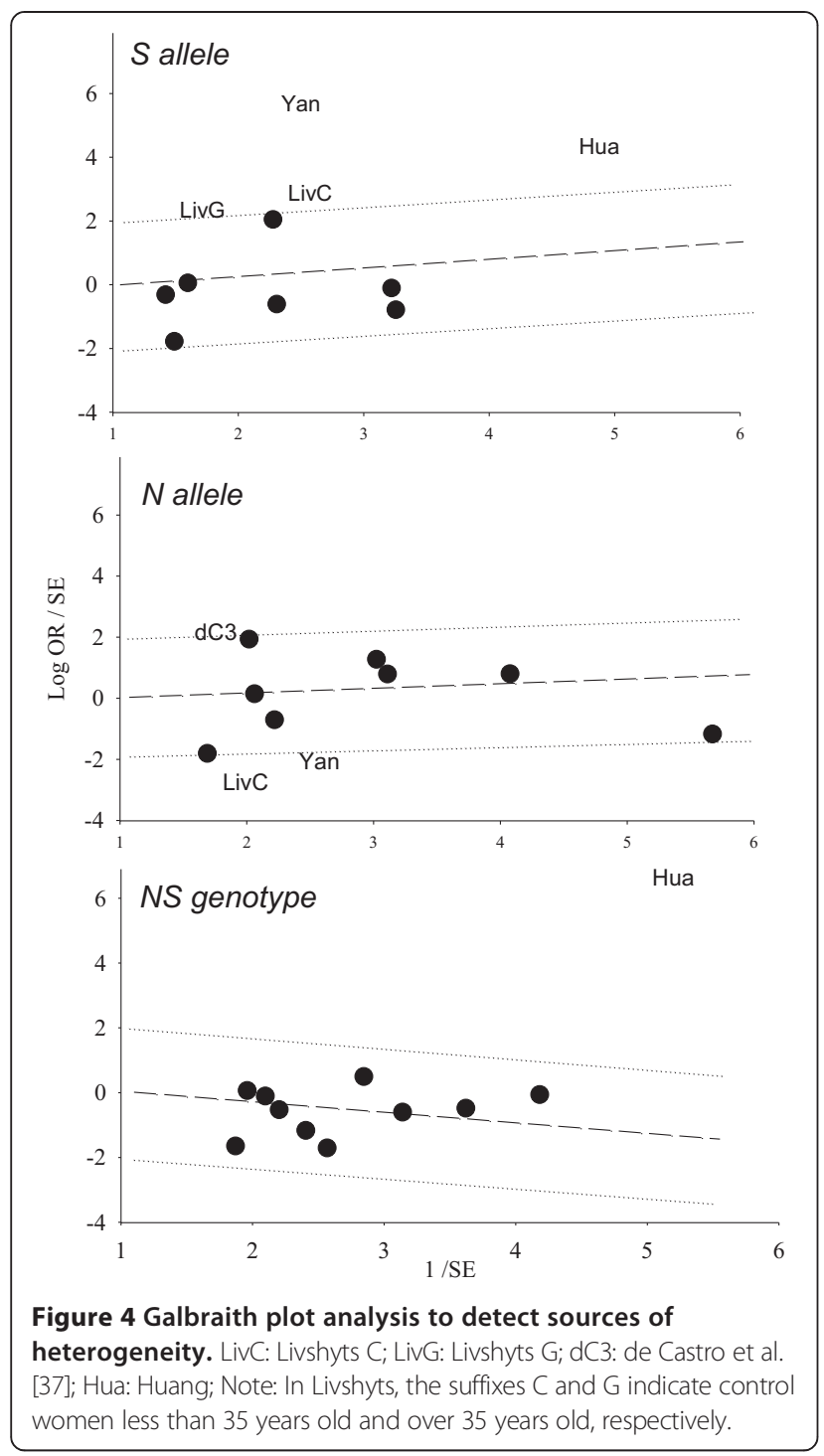

tendency to resist FSH stimulation and thus require more exogenous FSH for ovarian stimulation [45]. All these show a lack of consistency in these association studies outcomes. Here, then, enters the utility of our meta-analysis.

The observation that the allelic FSH receptor variants are FSH sensitivity determinants has lead to the hypothesis that certain genetic changes may fine tune the hormonal regulation of ovarian physiology, and these can be attractive markers for various clinical applications including optimization of the exogenous FSH dose in ART (Assisted Reproduction Technique) programs [20,46].

Approximately $20 \%$ of women undergoing ovarian stimulation in an IVF program respond poorly to gonadotropin treatment [47]. Such patients show low concentrations of serum estradiol, fewer mature oocytes and reduced pregnancy rates. The basis for low response to gonadotropin is not well understood. Diminished ovarian reserve and increased maternal age may be associated with poor ovarian response [6]. Several parameters such as poor perifollicular flow [48], presence of ovarian autoantibodies [49] and day 3 serum FSH concentrations [50,51] have been proposed as ovarian response predictors, but have not been proven. In some poor responders, increasing the FSH dose may not help in achieving an increase in serum estradiol concentrations [52].

Level of FSHR expression also impacts greatly on the extent of FSH action. FSHR plays a fundamental role in determining the physiologic FSH responsiveness in the ovary. Studies suggest that reduced expression affects FSHR function thereby affecting folliculogenesis [53]. The FSHR reduced expression on granulosa cells has been shown to be associated with poor ovarian response [54]. The expression of FSH receptor and its ability to respond to the exogenous FSH seems to be a gonadotropin determinant treatment. Thus, altered FSH receptor expression and function seems to be a factor and may account for poor ovarian response. Recently it has been demonstrated that lower expression of the FSH receptor may account for poor ovarian response to gonadotropin stimulation, suggesting a critical role of FSH receptor in the ovarian response [54].

Interpreting these meta-analysis results would warrant awareness of its strengths and limitations. Strengths include: (i) all tissue sources were blood; (ii) most of the studies addressed the HWE and LD issues; (iii) consistency in identifying the SS genotype as most likely to be associated with ovarian response. (iv) Since we did not include studies which controls deviated from the HWE, we managed to minimize methodological weakness, such as biased selection of subjects, genotyping errors and population stratification [55].

On the other hand, there are a number of limitations in our study: (i) we did not address age as covariate of poor and hyper-response given the insufficiency of primary data from the included studies. (ii) genotyping approaches were not uniform, although half of the studies used real-time PCR (Polymerase Chain Reaction).

It is possible that the N680S polymorphism does not play any direct functional role in the development of OHSS, but it is in LD with other polymorphisms. It is also likely that variants in other than FSHR are relevant to ovarian response [56]. Variants in genes relevant to biochemical pathways involved in steroids production and folliculogenesis have been studied, but replication of these relationships has been lacking $[13,57,58]$. Genome wide association studies may be promising as did a recent one that found significant correlation with ovarian response [59]. It is conceivable that ovarian response related to any one locus will be small due to gene-gene as well as gene-environment interactions are likely to operate. 
Table 3 Summary of sensitivity analysis findings

\begin{tabular}{|c|c|c|c|c|c|c|}
\hline \multicolumn{2}{|c|}{ Original summary effects } & \multirow[b]{2}{*}{ Nature of association } & \multirow[b]{2}{*}{ Omitted study [ref] } & \multicolumn{2}{|c|}{ Resulting pooled OR } & \multirow[b]{2}{*}{ Effect of study omission } \\
\hline OR & $95 \% \mathrm{Cl}$ & & & OR & $95 \% \mathrm{Cl}$ & \\
\hline \multicolumn{7}{|c|}{ NN genotype poor response overall } \\
\hline 0.95 & $0.66-1.37$ & no associations & Livshyts C [39] & 1.05 & $0.75-1.48$ & slight likelihood of $\mathrm{N}$ carriers being poor responders \\
\hline 0.95 & $0.66-1.37$ & no associations & Yan [41] & 1.03 & $0.70-1.50$ & slight likelihood of $\mathrm{N}$ carriers being poor responders \\
\hline \multicolumn{7}{|c|}{ SS genotype poor response NHC } \\
\hline 1.24 & $0.76-2.05$ & S carriers likely to be poor responders & Livshyts C [39] & 0.98 & $0.71-1.36$ & no associations \\
\hline \multicolumn{7}{|c|}{ NN genotype poor response NHC } \\
\hline 0.99 & $0.60-1.62$ & no associations & Livshyts C [39] & 1.23 & $0.93-1.64$ & likelihood of $\mathrm{N}$ carriers being poor responders \\
\hline 0.99 & $0.60-1.62$ & no associations & Livshyts G [39] & 1.12 & $0.69-1.82$ & likelihood of $\mathrm{N}$ carriers being poor responders \\
\hline \multicolumn{7}{|c|}{ NS genotype hyper-response NHC } \\
\hline 1.03 & $0.76-1.40$ & no associations & Daelemans [36] & 0.90 & $0.60-1.34$ & slight likelihood of NS carriers being non-hyper-responders \\
\hline
\end{tabular}




\section{Conclusion}

Genotyping the FSHR N680S together with some additional markers may provide a means of identifying a group of poor responders before infertility treatment is initiated. Meanwhile, further studies regarding other SNPs (or haplotypes) in the FSHR gene may help better understand the role of FSHR gene and ovarian response. Since potential biases and confounders could not be ruled out completely in this meta-analysis, additional large case-control studies or later update meta-analysis may be warranted to validate or modify our findings. It would help that well-designed studies based on sample sizes commensurate with detection of small genotypic risks should allow more definitive conclusions about the association of FSHR polymorphisms and ovarian response.

\section{Additional files}

Additional file 1: Table S1. Genotype frequencies of poor responders compared to normal/good responders. In Livshyts 2009, the suffixes C and $\mathrm{G}$ indicate control women under 35 years old and over 35 years old, respectively. In Boudjenah et al. [1], the suffixes $A$ and $S$ indicate overall population and homogeneous subgroup, respectively. NHC: Non-Hispanic Caucasian; HC: Hispanic Caucasian; maf: minor allele frequency; HWE: Hardy-Weinberg Equilibrium.

Additional file 2: Table S2. Genotype frequencies of hyper-responders compared to normal responders. In Boudjenah et al. [1], the suffixes A and S indicate overall population and homogeneous subgroup, respectively; NHC: Non-Hispanic Caucasian; HC: Hispanic Caucasian; maf: minor allele frequency; HWE: Hardy-Weinberg Equilibrium.

\section{Competing interests}

The authors declare that they have no competing interests.

\section{Authors contributions}

NP and BB conceived study design. NP, CMT, CP and HJ performed the data collection and analyzed data. NP, DMC, CPB and BB interpreted the data. All authors were involved in literature search, writing the paper and had final approval of the submitted and published versions.

\section{Acknowledgements}

This work was supported by FAPESP research grants \#2014/06177-2.

\section{Author details}

${ }^{1}$ Center for Research and Development, Angeles University Foundation, Angeles City 2009, Philippines. ${ }^{2}$ Graduate School, Cebu Doctors University, Mandaue City 6014, Philippines. ${ }^{3}$ Research and Extensions Office, Saint Louis University, BaguioCity 2006, Philippines. ${ }^{4}$ Human Reproduction and Genetics Center, Department of Collective Health - Faculdade de Medicina do ABC, Av. Prncipe de Gales, 821, Santo Andr/SP,

So Paulo Zip Code 09060-650, Brazil. ' ${ }^{5}$ Environmental Monitoring and Reporting Branch, Ontario Ministry of the Environment, 125 Resources Road, Etobicoke, ON M9P 3V6, Canada.

Received: 6 October 2014 Accepted: 11 December 2014 Published online: 20 December 2014

\section{References}

1. Boudjenah R, Molina-Gomes D, Torre A, Bergere M, Bailly M, Boitrelle F, Taieb S, Wainer R, Benahmed M, de Mazancourt P, Selva J, Vialard F: Genetic polymorphisms influence the ovarian response to rfsh stimulation in patients undergoing in vitro fertilization programs with icsi. PLoS One 2012, 7:e38700.
2. Mohiyiddeen L, Nardo LG: Single-nucleotide polymorphisms in the fsh receptor gene and ovarian performance: Future role in ivf. Human Fertil (Cambridge England) 2010, 13:72 78.

3. Sheikhha MH, Eftekhar M, Kalantar SM: Investigating the association between polymorphism of follicle-stimulating hormone receptor gene and ovarian response in controlled ovarian hyperstimulation. J Human Reprod Sci 2011, 4:86 90.

4. Huang X, Li L, Hong L, Zhou W, Shi H, Zhang H, Zhang Z, Sun X, Du J: The ser680asn polymorphism in the follicle-stimulating hormone receptor gene is associated with the ovarian response in controlled ovarian hyperstimulation. Clin Endocrinol (Oxf) 2014, doi: 10.1111/cen.12573. [Epub ahead of print]

5. Lledo B, Guerrero J, Turienzo A, Ortiz JA, Morales R, Ten J, Llacer J, Bernabeu R Effect of follicle-stimulating hormone receptor n680s polymorphism on the efficacy of follicle-stimulating hormone stimulation on donor ovarian response. Pharmacogenet Genomics 2013, 23:262 268.

6. Kligman I, Rosenwaks Z: Differentiating clinical profiles: Predicting good responders, poor responders, and hyperresponders. Fertil Steril 2001, 76:1185 1190.

7. Haller K, Salumets A, Uibo R: Anti-fsh antibodies associate with poor outcome of ovarian stimulation in ivf. Reprod Biomed Online 2008, 16:350 355

8. Freour T, Masson D, Mirallie S, Jean M, Bach K, Dejoie T, Barriere P: Active smoking compromises ivf outcome and affects ovarian reserve. Reprod Biomed Online 2008, 16:96 102.

9. Coccia ME, Rizzello F: Ovarian reserve. Ann N Y Acad Sci 2008, 1127:27 30

10. Altmae S, Haller K, Peters M, Hovatta O, Stavreus-Evers A, Karro H, Metspalu A, Salumets A: Allelic estrogen receptor 1 (esr1) gene variants predict the outcome of ovarian stimulation in in vitro fertilization. Mol Hum Reprod 2007, 13:521 526

11. Altmae S, Hovatta O, Stavreus-Evers A, Salumets A: Genetic predictors of controlled ovarian hyperstimulation: Where do we stand today? Hum Reprod Update 2011, 17:813 828

12. Moron FJ, Ruiz A: Pharmacogenetics of controlled ovarian hyperstimulation: Time to corroborate the clinical utility of fsh receptor genetic markers. Pharmacogenomics 2010, 11:1613 1618.

13. de Castro F, Moron FJ, Montoro L, Galan JJ, Hernandez DP, Padilla ES, Ramirez-Lorca R, Real LM, Ruiz A: Human controlled ovarian hyperstimulation outcome is a polygenic trait. Pharmacogenetics 2004, 14:285 293.

14. Gharib SD, Wierman ME, Shupnik MA, Chin WW: Molecular biology of the pituitary gonadotropins. Endocr Rev 1990, 11:177 199.

15. Simoni M, Gromoll J, Nieschlag E: The follicle-stimulating hormone receptor: Biochemistry, molecular biology, physiology, and pathophysiology. Endocr Rev 1997, 18:739 773.

16. Segaloff DL, Ascoli M: The lutropin/choriogonadotropin receptor 4 years later. Endocr Rev 1993, 14:324 347.

17. Gromoll J, Simoni M: Follicle-stimulating-hormone receptor and twinning. Lancet 2001, 357:230. author reply 231-232.

18. Aittomaki K, Lucena JL, Pakarinen P, Sistonen P, Tapanainen J, Gromoll J, Kaskikari R, Sankila EM, Lehvaslaiho H, Engel AR, Nieschlag E, Huhtaniemi I, de la Chapelle A: Mutation in the follicle-stimulating hormone receptor gene causes hereditary hypergonadotropic ovarian failure. Cell 1995, 82:959 968 .

19. Simoni M, Tempfer CB, Destenaves B, Fauser BC: Functional genetic polymorphisms and female reproductive disorders: Part i: Polycystic ovary syndrome and ovarian response. Hum Reprod Update 2008, 14:459 484.

20. Perez Mayorga M, Gromoll J, Behre HM, Gassner C, Nieschlag E, Simoni M: Ovarian response to follicle-stimulating hormone (fsh) stimulation depends on the fsh receptor genotype. J Clin Endocrinol Metab 2000, 85:3365 3369 .

21. Simoni M, Nieschlag E, Gromoll J: Isoforms and single nucleotide polymorphisms of the fsh receptor gene: Implications for human reproduction. Hum Reprod Update 2002, 8:413 421.

22. Yao Y, Ma CH, Tang HL, Hu YF: Influence of follicle-stimulating hormone receptor (fshr) ser680asn polymorphism on ovarian function and in-vitro fertilization outcome: A meta-analysis. Mol Genet Metab 2011, 103:388 393.

23. Laan M, Grigorova M, Huhtaniemi IT: Pharmacogenetics of follicle-stimulating hormone action. Current Opin Endocrinol Diabetes Obesity 2012, 19:220 227.

24. Kene PS, Nalavadi VC, Dighe RR, lyer KS, Mahale SD: Identification of the structural and functional determinants of the extracellular domain of the 
human follicle stimulating hormone receptor. J Endocrinol 2004, 182:501 508 .

25. Clark MF, Baudouin SV: A systematic review of the quality of genetic association studies in human sepsis. Intensive Care Med 2006, 32:1706 1712.

26. Mantel N, Haenszel W: Statistical aspects of the analysis of data from retrospective studies of disease. J Natl Cancer Inst 1959, 22:719 748.

27. DerSimonian R, Laird N: Meta-analysis in clinical trials. Control Clin Trials 1986, 7:177 188.

28. Lau J, loannidis JP, Schmid CH: Quantitative synthesis in systematic reviews. Ann Intern Med 1997, 127:820 826.

29. Higgins JP, Thompson SG, Deeks JJ, Altman DG: Measuring inconsistency in meta-analyses. BMJ Clin Res 2003, 327:557 560.

30. Higgins JP, Thompson SG: Quantifying heterogeneity in a meta-analysis. Stat Med 2002, 21:1539 1558

31. Ioannidis JP, Trikalinos TA: The appropriateness of asymmetry tests for publication bias in meta-analyses: A large survey. Cmaj 2007, 176:1091 1096

32. Begg $\mathrm{CB}$, Mazumdar M: Operating characteristics of a rank correlation test for publication bias. Biometrics 1994, 50:1088 1101.

33. Moher D, Liberati A, Tetzlaff J, Altman DG: Preferred reporting items for systematic reviews and meta-analyses: The prisma statement. PLoS Med 2009, 6:e1000097.

34. Loutradis D, Patsoula E, Minas V, Koussidis GA, Antsaklis A, Michalas S, Makrigiannakis A: Fsh receptor gene polymorphisms have a role for different ovarian response to stimulation in patients entering ivf/icsi-et programs. J Assist Reprod Genet 2006, 23:177 184.

35. Achrekar SK, Modi DN, Desai SK, Mangoli VS, Mangoli RV, Mahale SD: Follicle-stimulating hormone receptor polymorphism (thr307ala) is associated with variable ovarian response and ovarian hyperstimulation syndrome in indian women. Fertil Steril 2009, 91:432 439.

36. Daelemans C, Smits G, de Maertelaer V, Costagliola S, Englert Y, Vassart G, Delbaere A: Prediction of severity of symptoms in iatrogenic ovarian hyperstimulation syndrome by follicle-stimulating hormone receptor ser680asn polymorphism. J Clin Endocrinol Metab 2004, 89:6310 6315.

37. de Castro F, Ruiz R, Montoro L, Perez-Hernandez D, Sanchez-Casas Padilla E, Real LM, Ruiz A: Role of follicle-stimulating hormone receptor ser680asn polymorphism in the efficacy of follicle-stimulating hormone. Fertil Steril 2003, 80:571 576

38. Klinkert ER, te Velde ER, Weima S, van Zandvoort PM, Hanssen RG, Nilsson PR, de Jong FH, Looman CW, Broekmans FJ: Fsh receptor genotype is associated with pregnancy but not with ovarian response in ivf. Reprod Biomed Online 2006, 13:687 695.

39. Livshyts G, Podlesnaja S, Kravchenko S, Sudoma I, Livshits L: A distribution of two snps in exon 10 of the fshr gene among the women with a diminished ovarian reserve in ukraine. J Assist Reprod Genet 2009, 26:29 34

40. Mohiyiddeen L, Newman WG, Cerra C, McBurney H, Mulugeta B, Roberts SA, Nardo LG: A common asn680ser polymorphism in the follicle-stimulating hormone receptor gene is not associated with ovarian response to gonadotropin stimulation in patients undergoing in vitro fertilization. Fertil Steril 2013, 99:149 155.

41. Yan Y, Gong Z, Zhang L, Li Y, Li X, Zhu L, Sun L: Association of follicle-stimulating hormone receptor polymorphisms with ovarian response in chinese women: A prospective clinical study. PLoS One 2013, 8:e78138.

42. Binder H, Strick R, Zaherdoust O, Dittrich R, Hamori M, Beckmann MW, Oppelt PG: Assessment of fshr variants and antimullerian hormone in infertility patients with a reduced ovarian response to gonadotropin stimulation. Fertil Steril 2012, 97:1169 1175. e1161.

43. Jun JK, Yoon JS, Ku SY, Choi YM, Hwang KR, Park SY, Lee GH, Lee WD, Kim SH, Kim JG, Moon SY: Follicle-stimulating hormone receptor gene polymorphism and ovarian responses to controlled ovarian hyperstimulation for ivf-et. J Hum Genet 2006, 51:665 670 .

44. La Marca A, Sighinolfi G, Argento C, Grisendi V, Casarini L, Volpe A, Simoni $M$ : Polymorphisms in gonadotropin and gonadotropin receptor genes as markers of ovarian reserve and response in in vitro fertilization. Fertil Steril 2013, 99:970 978, e971.

45. Loutradis D, Theofanakis C, Anagnostou E, Mavrogianni D, Partsinevelos GA: Genetic profile of snp(s) and ovulation induction. Curr Pharm Biotechnol 2012, 13:417 425.

46. Behre HM, Greb RR, Mempel A, Sonntag B, Kiesel L, Kaltwasser P, Seliger E, Ropke F, Gromoll J, Nieschlag E, Simoni M: Significance of a common single nucleotide polymorphism in exon 10 of the follicle-stimulating hormone (fsh) receptor gene for the ovarian response to fsh: A pharmacogenetic approach to controlled ovarian hyperstimulation. Pharmacogenet Genomics 2005, 15:451 456

47. Keay SD, Liversedge NH, Mathur RS, Jenkins JM: Assisted conception following poor ovarian response to gonadotrophin stimulation. Br J Obstet Gynaecol 1997, 104:521 527.

48. Battaglia C, Genazzani AD, Regnani G, Primavera MR, Petraglia F, Volpe A: Perifollicular doppler flow and follicular fluid vascular endothelial growth factor concentrations in poor responders. Fertil Steril 2000, 74:809 812.

49. Luborsky UL, Thiruppathi P, Rivnay B, Roussev R, Coulam C, Radwanska E: Evidence for different aetiologies of low estradiol response to fsh: Age-related accelerated luteinization of follicles or presence of ovarian autoantibodies. Human Reprod (Oxford England) 2002, 17:2641 2649.

50. Balasch J, Creus M, Fabreques F, Carmona F, Casamitjana R, Ascaso C, Vanrell JA: Inhibin, follicle-stimulating hormone, and age as predictors of ovarian response in in vitro fertilization cycles stimulated with gonadotropin-releasing hormone agonist-gonadotropin treatment. Am J Obstet Gynecol 1996, 175:1226 1230

51. Barnhart K, Osheroff J: Follicle stimulating hormone as a predictor of fertility. Current Opin Obstetrics Gynecol 1998, 10:227 232

52. Land JA, Yarmolinskaya MI, Dumoulin JC, Evers JL: High-dose human menopausal gonadotropin stimulation in poor responders does not improve in vitro fertilization outcome. Fertil Steril 1996, 65:961 965.

53. Oktay K, Briggs D, Gosden RG: Ontogeny of follicle-stimulating hormone receptor gene expression in isolated human ovarian follicles. J Clin Endocrinol Metab 1997, 82:3748 3751.

54. Cai J, Lou HY, Dong MY, Lu XE, Zhu YM, Gao HJ, Huang HF: Poor ovarian response to gonadotropin stimulation is associated with low expression of follicle-stimulating hormone receptor in granulosa cells. Fertil Steril 2007, 87:1350 1356.

55. Thakkinstian A, McElduff P, D'Este C, Duffy D, Attia J: A method for meta-analysis of molecular association studies. Stat Med 2005, 24:1291 1306

56. Loutradis D, Vlismas A, Drakakis $\mathrm{P}$, Antsaklis A: Pharmacogenetics in ovarian stimulation current concepts. Ann N Y Acad Sci 2008, 1127:10 19.

57. Kevenaar ME, Themmen AP, Laven JS, Sonntag B, Fong SL, Uitterlinden AG, De Jong FH, Pols HA, Simoni M, Visser JA: Anti-mullerian hormone and anti-mullerian hormone type ii receptor polymorphisms are associated with follicular phase estradiol levels in normo-ovulatory women. Human Reprod (Oxford England) 2007, 22:1547 1554.

58. Moron FJ, de Castro F, Royo JL, Montoro L, Mira E, Saez ME, Real LM, Gonzalez A, Manes S, Ruiz A: Bone morphogenetic protein 15 (bmp15) alleles predict over-response to recombinant follicle stimulation hormone and iatrogenic ovarian hyperstimulation syndrome (ohss). Pharmacogenet Genomics 2006, 16:485 495 .

59. van Disseldorp J, Franke L, Eijkemans R, Broekmans F, Macklon N, Wijmenga C, Fauser B: Genome-wide analysis shows no genomic predictors of ovarian response to stimulation by exogenous fsh for ivf. Reprod Biomed Online 2011, 22:382 388 .

doi:10.1186/s13048-014-0122-2

Cite this article as: Pabalan et al:: Evaluating influence of the genotypes in the follicle-stimulating hormone receptor (FSHR) Ser680Asn (rs6166) polymorphism on poor and hyper-responders to ovarian stimulation: a meta-analysis. Journal of Ovarian Research 2014 7:122. 